\title{
Deep optical observations of the supernova remnant G 78.2+2.1
}

\author{
F. Mavromatakis ${ }^{\star}$ \\ University of Crete, Physics Department, PO Box 2208, 71003 Heraklion, Crete, Greece \\ Received 7 May 2003 / Accepted 12 June 2003

\begin{abstract}
The wide-field covered by the supernova remnant G 78.2+2.1 was observed in the optical emission lines of $\mathrm{H} \alpha+$ [N II], [S II] and [O III]. The flux calibrated images reveal several H II regions in the field which dominate the optical emission but we were able to identify possible areas of shock-heated emission through the $\mathrm{H} \alpha+[\mathrm{N}$ II] and [S II] images. These are mainly found to the north-east of $\gamma$ Cygni as well as in the south and the morphology of the detected emission is patchy and diffuse. A few patchy structures are also detected in the medium ionization line image of [O III]. Long-slit spectra taken at one of the candidate positions verify that we have detected radiation from shock-heated gas ([S II $] / \mathrm{H} \alpha \simeq 0.6$ ). The estimated shock velocity lies below $100 \mathrm{~km} \mathrm{~s}^{-1}$, while the measured electron density of $\sim 700 \mathrm{~cm}^{-3}$ implies preshock cloud densities of $\sim 20 \mathrm{~cm}^{-3}$. High resolution maps in the infrared show that the optical emission, which may be associated with $\mathrm{G} 78.2+2.1$, lies in areas relatively free of infrared emission. The interstellar extinction measured through the optical spectra is compatible with current estimates of the distance to the remnant. The optical data are in agreement with the explosion energy and interstellar medium density estimated from the X-ray data suggesting that the remnant is still in the adiabatic phase of its evolution. A second set of spectra taken in the north-west suggests that we are probably dealing with a foreground $\mathrm{H}$ II region.
\end{abstract}

Key words. ISM: general - ISM: supernova remnants - ISM: individual objects: G 78.2+2.1

\section{Introduction}

Optical observations of supernova remnants can provide us information about the properties of the interstellar medium (ISM) and, under certain assumptions, about the properties of the remnant itself (e.g. McKee \& Cowie 1975). The ISM is known to be neither homogeneous nor uniform encompassing dense concentrations of gas known as interstellar "clouds". The interaction of the primary shock wave with these "clouds" leads to effects observable at optical wavelengths.

The supernova remnant G 78.2+2.1 lies in the complex area of Cygnus where many bright and dark nebulae are found. The first radio and optical observations focused on the emission in the neighborhood of the bright star $\gamma$ Cygni (e.g. Johnson 1974; d'Odorico \& Sabbadin 1977; Bohigas et al. 1983). However, Higgs et al. (1977) using radio maps at $1.4 \mathrm{GHz}$ and $10 \mathrm{GHz}$ were able to identify a $\sim 62^{\prime}$ diameter shell and estimated a non-thermal spectral index $\alpha$ of $0.7 \pm 0.1\left(S_{v} \sim v^{-a}\right)$. These radio maps, as well as newer maps at various radio frequencies, show that the most intense radiation is found in the south-east (close to $\gamma$ Cygni) and in the north-west (e.g. Zhang et al. 1997; Pineault \& Chastenay 1990). Zhang et al. (1997) combined data from different frequencies to determine an integrated spectral index of $0.54 \pm 0.02$ and also detected systematic spatial variations of the index of the order of \pm 0.15 . However, the origin of these variations remains an open issue. The distance to the remnant is estimated by

\footnotetext{
* e-mail: fotis@physics.uoc.gr
}

Landecker et al. (1980), using a number of arguments, as $1.5 \pm 0.5 \mathrm{kpc}$. At this distance the shell is $\sim 55 \mathrm{pc}$ above the galactic plane and its radius is $\sim 14 \mathrm{pc}$. Lozinskaya et al. (2000) performed interferometric observations in $\mathrm{H} \alpha$ and [N II] in selected areas inside and outside the extent of the remnant in an attempt to study its radial-velocity field. Nevertheless, the authors, based on the kinematic data, could not exclude the possibility that the optical nebulae towards G $78.2+2.1$ are H II regions. They also analyzed archival ROSAT and ASCA data from this area of the sky and proposed that the remnant is in the adiabatic phase of its evolution. Uchiyama et al. (2002) analyzed the same ASCA data and found several different spectral components. Although the details differ, both analysis suggest shock velocities around $10^{3} \mathrm{~km} \mathrm{~s}^{-1}$ and an age less than $10^{4} \mathrm{yr}$. $\mathrm{G} 78.2+2.1$ is an exciting object because of its proximity to the strong $\gamma$-ray source 2EG J2020+4026 (Esposito et al. 1996), while Brazier et al. (1996) detected an X-ray source within the $\gamma$-ray error circle. The $\gamma$-ray and X-ray properties of the emission lead the latter authors to propose that it originates from a pulsar that could be associated with the remnant.

Very little information is known about the optical properties of G 78.2+2.1. Currently, there do not exist in the literature dedicated optical observations of this remnant with the exception of the survey of Parker et al. (1979), while van den Bergh (1978) observed only a narrow field in the vicinity of $\gamma$ Cygni. In addition, its position on the celestial sphere makes it an ideal target to observe from our site. In this work we present the first flux calibrated CCD images of the whole field of G 78.2+2.1 in 
Table 1. Imaging log.

\begin{tabular}{lll}
\hline \hline $\mathrm{H} \alpha+[\mathrm{N} \mathrm{II}]$ & {$[\mathrm{S} \mathrm{II}]$} & {$[\mathrm{O} \mathrm{III}]$} \\
\hline $4800^{a}(2)^{b}$ & $4800(2)$ & $7200(3)$ \\
\hline $6560^{c} \AA(75 \AA)^{d}$ & $6708 \AA(20 \AA)$ & $5005 \AA(28 \AA)$ \\
\hline${ }^{a}$ Total exposure time in s. \\
${ }^{b}$ Number of individual frames. \\
${ }^{c}$ Central wavelength. \\
${ }^{d}$ Full width at half maximum.
\end{tabular}

the emission lines of $\mathrm{H} \alpha+[\mathrm{N} \mathrm{II}]$, [S II] and [O III]. Deep longslit spectra in two areas were also acquired. Information about the observations and the data reduction is given in Sect. 2. In Sects. 3 and 4 the results of the imaging and spectral observations are presented, while in Sect. 5 the properties of the remnant and its environment are discussed. Finally, in Sect. 6 we summarize the results of this work.

\section{Observations}

\subsection{Optical images}

The imaging observations were performed with the $0.3 \mathrm{~m}$ widefield Schmidt-Cassegrain telescope at Skinakas Observatory, Crete, Greece. The field around G 78.2+2.1 was observed on August 7 and 8, 2002. The telescope was equipped with a $1024 \times 1024$ Thomson CCD providing a $70^{\prime} \times 70^{\prime}$ field of view and an image scale of 4 " per pixel. The remnant was observed through the $\mathrm{H} \alpha+[\mathrm{N} \mathrm{II}]$, [S II], and [O III] filters. Details on the filters used and the exposure times can be found in Table 1. An astrometric solution was derived for each data frame using the HST Guide star catalogue (Lasker et al. 1999). All images were subsequently projected to a common origin on the sky for further operations. The equatorial coordinates quoted in this work refer to epoch 2000.

The data were reduced using standard IRAF and MIDAS routines. The available frames were bias subtracted and flatfield corrected using a series of well exposed twilight flatfields. The absolute flux calibration was performed through observations of a series of spectrophotometric standard stars (HR 5501, HR 7596, HR 7950, HR 9087, HR 718, and HR 8634; Hamuy et al. 1992, 1994).

\subsection{Optical spectra}

Long-slit spectra in the area of the remnant were obtained on August 9, 10 and 11, 2002 with the $1.3 \mathrm{~m}$ Ritchey-Cretien telescope at Skinakas Observatory. The data were acquired with a 1300 line $\mathrm{mm}^{-1}$ grating and a $800 \times 2000$ SITe CCD covering the range of $4750 \AA-6815 \AA$. The slit width was 7.'7, the slit length was 7.9 and it was always oriented in the south-north direction. The coordinates of the slit centers, the number of available spectra from each location and the total exposure times are given in Table 3. The spectrophotometric standard stars HR 7596, HR 7950, and HR 9087, HR 718 and HR 8634 were observed for the absolute calibration of the available spectra.

\section{The imaging observations}

\subsection{The $\mathrm{H} \alpha+[\mathrm{NII}]$ and S II line images}

The low-ionization emission in $\mathrm{H} \alpha+[\mathrm{NII}]$ from the area of G 78.2+2.1 is shown in Fig. 1. The bright nebulae dominate the emission in this field, while dark nebulae mark their presence through absorption of background emission. The open cluster NGC 6910 lies in the north-east area of the image, while the $2.2 \mathrm{mag}$ star $\gamma$ Cygni in the south-east is heavily overexposed in these deep exposures. Filamentary structures are not immediately seen in this complex field, instead patches of bright emission are present in the north-west and the east. In Fig. 1 we also overlay the radio-continuum contours at $1420 \mathrm{MHz}$ from the Canadian Galactic Plane Survey (CGPS - Taylor et al. 2003) and discover that the patchy optical emission shows some degree of correlation with the radio emission. It is not clear if the radio emission at the correlation areas is non-thermal or thermal, i.e. if the corresponding optical emission is due to shock-heating or photoionization. Sulfur line emission from H II regions is generally suppressed compared to emission from a shock-heated gas. The [S II] image, shown in Fig. 2, displays the same overall morphology as the $\mathrm{H} \alpha+[\mathrm{N} \mathrm{II}]$ image, however certain areas emit less intense sulfur line radiation. In an attempt to identify shock-heated emission, we use the ratio of these two low-ionization images since they are flux calibrated. The $\mathrm{H} \alpha+[\mathrm{N} \mathrm{II}]$ filter transmits equally well the $\mathrm{H} \alpha$ and $\left[\mathrm{N}_{\mathrm{II}}\right] 6548,6584 \AA$ lines, while the sulfur filter transmits $100 \%$ of the $6716 \AA$ line and $18 \%$ of the $6731 \AA$ line. Assuming the ratio of these two latter lines to be $\sim 1$ (see Sect. 4) and that the $\mathrm{H} \alpha$ flux is $\sim 1-1.5$ of the [N II] flux, we find that $([\mathrm{S} \mathrm{II}] / \mathrm{H} \alpha)_{\text {intrinsic }} \sim 3([\mathrm{~S} \mathrm{II}] / \mathrm{H} \alpha+[\mathrm{N} \mathrm{II}])_{\text {observed. The }}$ bulk of the emission in the west, north-west seems to originate from photoionized gas since a ratio of $0.33( \pm 0.04)$ is measured (e.g. Hunter et al. 1992), while in the south, around $\alpha \sim 20^{\mathrm{h}} 20^{\mathrm{m}}, \delta \sim 40^{\circ} 00^{\mathrm{m}}$, we find a ratio of $0.59( \pm 0.03$; Smith et al. 1993). The bright emission is the east is mainly due to photoionization. However, there are specific locations where ratios of $0.5-0.7$ are measured. One of those coincides with the location where a long-slit spectrum was taken and the more accurately measured [S II] $/ \mathrm{H} \alpha$ ratio turns out to be 0.65 (Table 2). Another structure easily identified in the [S II] image is found around $\alpha \simeq 20^{\mathrm{h}} 23^{\mathrm{m}} 20^{\mathrm{s}}$ and $\delta \simeq 40^{\circ} 27^{\prime}$, at an average flux level of $25 \times 10^{-17} \mathrm{erg} \mathrm{s}^{-1} \mathrm{~cm}^{-2} \operatorname{arcsec}^{-2}$. Interestingly, this source appears less prominent in the $\mathrm{H} \alpha+[\mathrm{N} \mathrm{II}]$ image due to the ambient patchy emission which is almost as strong. The $[\mathrm{S} \mathrm{II}] / \mathrm{H} \alpha$ ratios measured along this structure are in the range of $0.5-0.6$ suggesting that the observed emission can have a shock-heated origin. It is evident that the use of the flux calibrated images provides rough estimates of the $[\mathrm{S} \mathrm{II}] / \mathrm{H} \alpha$ ratio but it does show that it may vary by a factor of 2 within the field of the remnant. In Fig. 2 the contours of infrared emission (IR) at $8.28 \mu \mathrm{m}$ are overlaid to the [S II] image. These show that the locations with high $[\mathrm{S} \mathrm{II}] / \mathrm{H} \alpha$ ratios are found in areas free of IR emission or with weak IR emission.

\subsection{The [O III] image}

The medium ionization line of $[\mathrm{O}$ III] $5007 \AA$ provides a better view of the area, compared to the lower ionization 


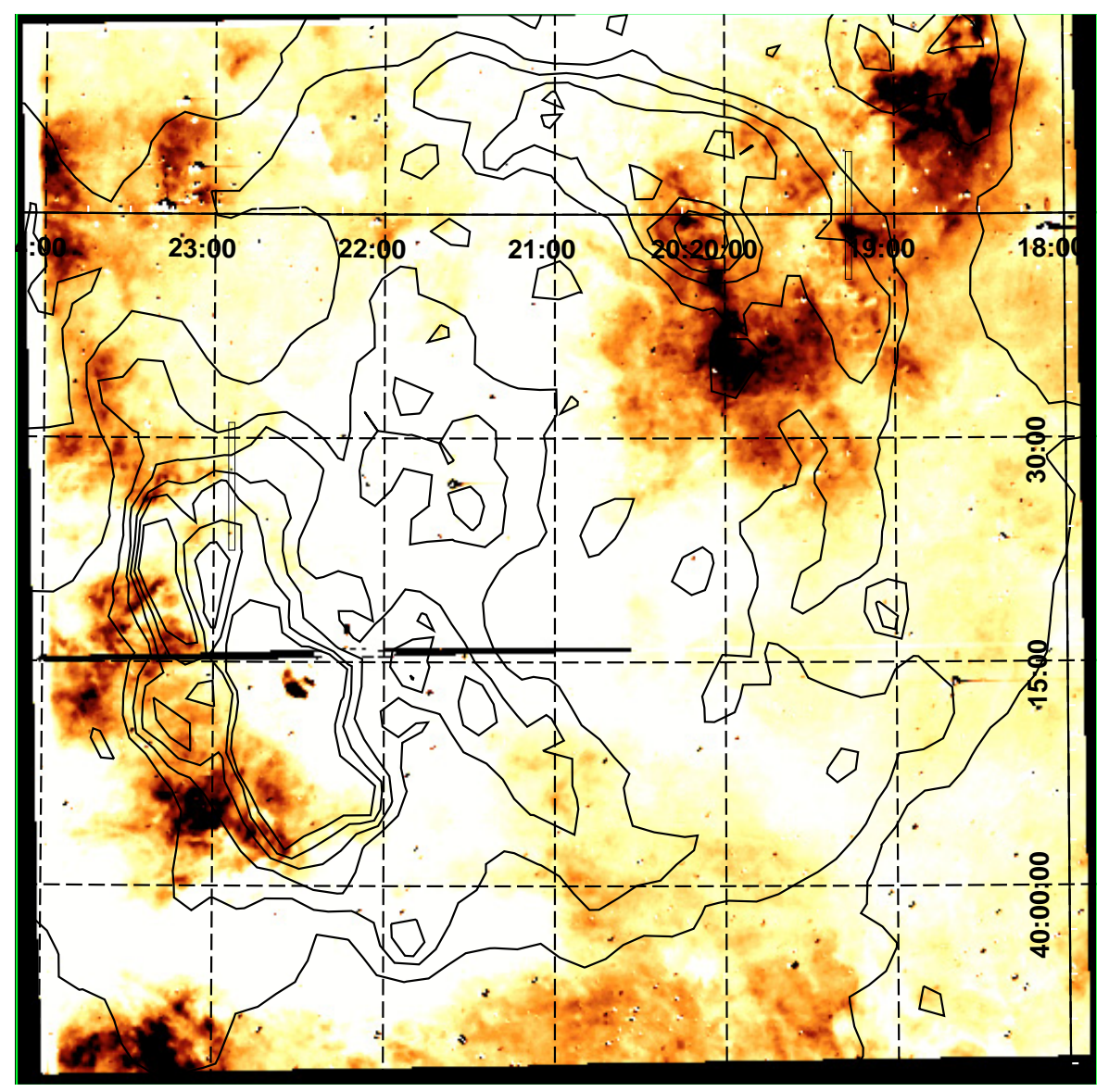

Fig. 1. Diffuse and patchy emission dominates the field of G 78.2+2.1. The shadings run linearly from 0 to $280 \times 10^{-17} \mathrm{erg} \mathrm{s}^{-1} \mathrm{~cm}^{-2} \mathrm{arcsec}^{-2}$, while the $1400 \mathrm{MHz}$ contours scale also linearly from 5 to $90 \mathrm{~K}$ brightness temperature in steps of $9.3 \mathrm{~K}$ (Taylor et al. 2003). The long rectangles in the east and north-west mark the slit projections on the sky. The line segments seen near over-exposed stars in this figure and the next figures are due to the blooming effect. North is up, East is to the left and the coordinates given in this figure and the next figures refer to epoch $\mathbf{J} 2000$.

images, since H II regions are not particularly strong in this line (Fig. 3). In general, the patchy structures in the north-west are also present in [O III], as well as the diffuse emission in the south. A filamentary structure in the north-west seems correlated with the $1400 \mathrm{MHz}$ emission but we can not verify its association with the remnant on the basis of the $[\mathrm{S} \mathrm{II}] / \mathrm{H} \alpha$ ratio. Relatively strong [O III] emission is present to the south-east and to the north-east of $\gamma$ Cygni. The structure detected in the [S II] image at $\alpha \simeq 20^{\mathrm{h}} 23^{\mathrm{m}} 20^{\mathrm{s}}$ and $\delta \simeq 40^{\circ} 27^{\prime}$ is also quite prominent in this line and nicely aligned along the radio contours. Its angular length is $\sim 4^{\prime}$, while the measured full width at half maximum of its intensity is $30^{\prime \prime}$. The absolute flux level of $15-20 \times 10^{-17} \mathrm{erg} \mathrm{s}^{-1} \mathrm{~cm}^{-2} \operatorname{arcsec}^{-2}$ when compared with its $\mathrm{H} \alpha+[\mathrm{N}$ II $]$ flux lead us to conclude that the recombination zone behind the shock is complete (e.g. Raymond et al. 1988) even if this radiation is heavily absorbed (Sect. 4). Of course, this conclusion applies in the case where the detected emission originates from shock-heated gas. Dedicated optical spectroscopic observations would easily resolve this issue and establish its relation to $\mathrm{G} 78.2+2.1$. Weak filamentary emission is also present around $\alpha \simeq 20^{\mathrm{h}} 23^{\mathrm{m}} 39^{\mathrm{s}}$ and $\delta \simeq 40^{\circ} 31^{\prime} 20^{\prime \prime}$ and displays the same orientation as the outermost radio contours at $1400 \mathrm{MHz}$.

\section{The long-slit spectra from $G$ 78.2+2.1}

Given the available observing time, two sets of deep long-slit spectra were obtained from the area $G 78.2+2.1$. The positions were chosen by examining the [S II] image and requiring that the optical emission lies within the radio boundary, no stars are present in the target area and local background subtraction is possible. The first set of spectra was taken in the north-west (pos. 1), while the second one was taken in the east (pos. 2). The projections of the slits on the sky are shown in Fig. 1 as long rectangles.

We were able to extract four different apertures from pos. 1 . All of these spectra suggest that the emitting gas is of photoionized origin ([S II $] / \mathrm{H} \alpha \sim 0.2-0.3$; Table 2). In this table we list only one of the extracted spectra, that from the brightest patch of emission at $\alpha \simeq 20^{\mathrm{h}} 19^{\mathrm{m}} 15^{\mathrm{s}}$ and $\delta \simeq 40^{\circ} 40^{\prime} 44^{\prime \prime}$. The electron density is calculated with the temden task of the nebular package (Shaw \& Dufour 1995) and is found to be $\sim 630 \mathrm{~cm}^{-3}$. The [O III] $5007 \AA$ flux is quite weak amounting to only $50 \%$ of the $\mathrm{H} \beta$ flux. Two apertures were extracted from pos. 2 and both are listed in Table 2 . One of them displays the characteristic signature of emission from shock-heated gas ([S II] $/ \mathrm{H} \alpha \sim$ 0.6 ), while the second seems to point to emission from photoionized gas $([\mathrm{S}$ II $] / \mathrm{H} \alpha \sim 0.2)$. The electron density in the 


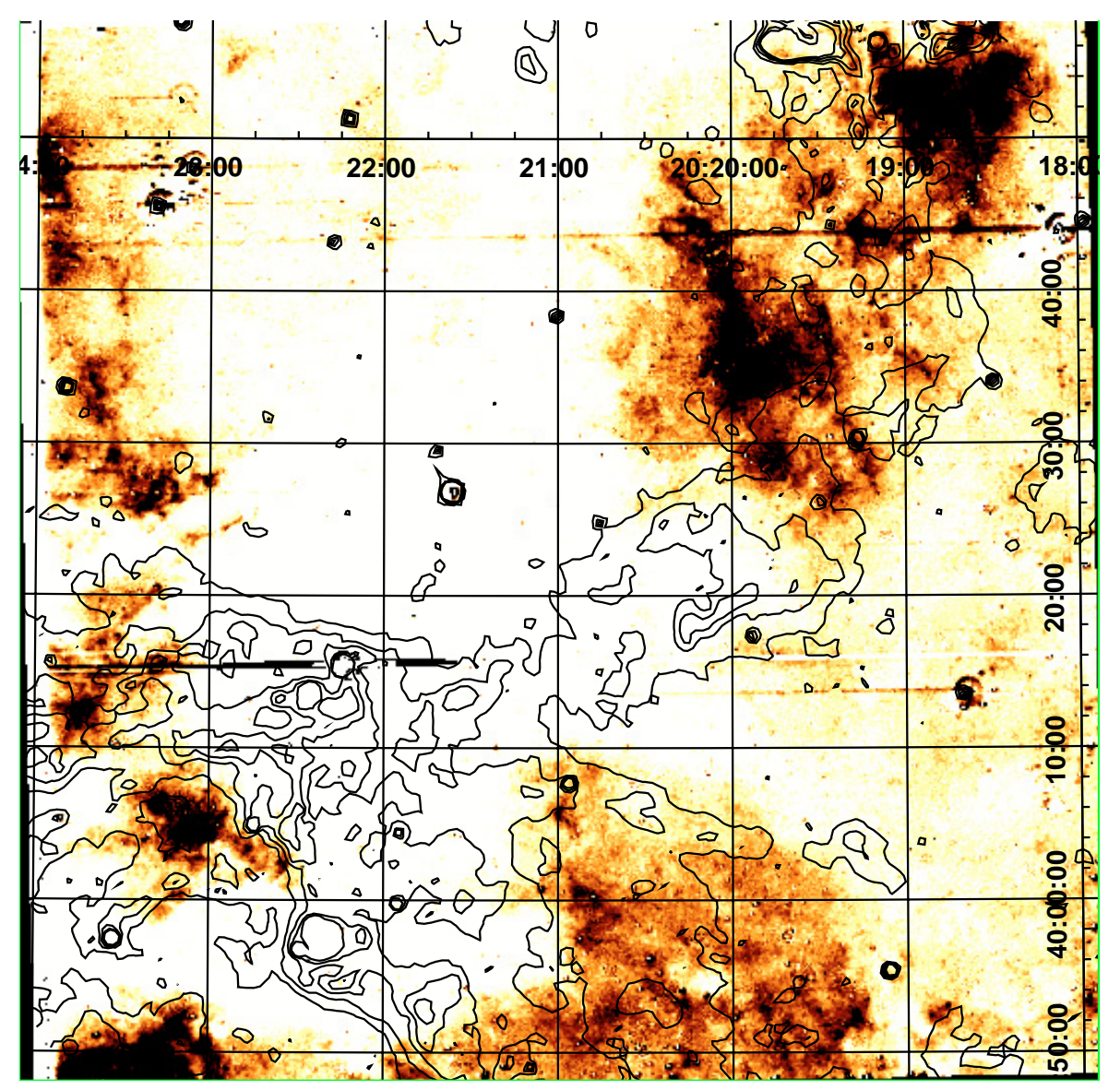

Fig. 2. The same field as in Fig. 1 but now seen through the sulfur filter. The shadings run linearly from 0 to $25 \times 10^{-17} \mathrm{erg} \mathrm{s}^{-1} \mathrm{~cm}^{-2} \operatorname{arcsec}^{-2}$, while the infrared emission at $8.28 \mu \mathrm{m}$ is shown in contours scaling from $8 \times 10^{-7}$ to $8 \times 10^{-6} \mathrm{~W} \mathrm{~m}^{-2} \mathrm{sr}^{-1}$. Evidence for shock-heated gas are not present in areas of strong IR emission.

Table 2. Relative line fluxes.

\begin{tabular}{lllllll}
\hline \hline & Area Ia & Area Ib & Area IIa & Area IIb & Area IIc & Area IId \\
\hline Line $(\AA)$ & $F^{a, b}$ & $F^{a, b}$ & $F^{a, b}$ & $F^{a, b}$ & $F^{a, b}$ & \\
\hline $4861 \mathrm{H} \beta$ & $9.8(6)$ & $14.4(6)$ & $17.9(19)$ & $15.8(28)$ & $13.4(23)$ & $18.5(25)$ \\
\hline $4959[\mathrm{O} \mathrm{III}]_{1}$ & - & - & $2.6(3)$ & $2.1(4)$ & $0.9(2)$ & $1.8(2)$ \\
\hline $5007[\mathrm{O} \mathrm{III}]_{2}$ & $4.9(3)$ & $8(3)$ & $10.2(12)$ & $7.0(13)$ & $3.8(7)$ & $6.4(10)$ \\
\hline $5876 \mathrm{He} \mathrm{I}$ & - & - & $2.8(6)$ & $2.7(11)$ & $2.3(8)$ & $2.0(7)$ \\
\hline $6300[\mathrm{OI}]_{1}$ & $3.8(2)$ & - & $2.1(5)$ & $0.9(5)$ & $0.7(3)$ & $2.7(12)$ \\
\hline $6364[\mathrm{O} \mathrm{I}]_{2}$ & - & - & - & - & - & $0.7(3)$ \\
\hline $6548[\mathrm{~N} \mathrm{II}]_{1}$ & $9.4(12)$ & $5.0(3)$ & $12.2(27)$ & $11.9(52)$ & $11.7(47)$ & $12.7(44)$ \\
\hline $6563 \mathrm{H} \alpha$ & $100(122)$ & $100(68)$ & $100(207)$ & $100(376)$ & $100(354)$ & $100(293)$ \\
\hline $6584[\mathrm{~N} \mathrm{II}]_{2}$ & $53.7(66)$ & $39.4(27)$ & $44.6(97)$ & $39.7(168)$ & $43.3(165)$ & $45.7(135)$ \\
\hline $6716[\mathrm{~S} \mathrm{II}]_{1}$ & $29.6(42)$ & $13.2(10)$ & $13.4(32)$ & $11.5(52)$ & $13.8(58)$ & $16.5(57)$ \\
\hline $6731[\mathrm{~S} \mathrm{II}]_{2}$ & $31.2(44)$ & $11.2(9)$ & $12.2(29)$ & $11.9(54)$ & $13.9(58)$ & $16.7(57)$ \\
\hline \hline $\mathrm{Absolute} \mathrm{H} \alpha$ flux ${ }^{c}$ & 56 & 50 & 81 & 149 & 239 & 62 \\
\hline $\mathrm{H} \alpha / \mathrm{H} \beta$ & $10.2(6)$ & $6.9(6)$ & $5.6(19)$ & $6.3(28)$ & $7.5(23)$ & $5.4(25)$ \\
\hline$c$ & $1.6[0.2]$ & $1.1[0.2]$ & $0.82[0.07]$ & $0.97[0.05]$ & $1.20[0.06]$ & $0.77[0.05]$ \\
\hline$[\mathrm{O} \mathrm{III]}] / \mathrm{H} \beta$ & $0.7(3)$ & - & $0.76(10)$ & $0.59(12)$ & $0.38(7)$ & $0.46(9)$ \\
\hline$[\mathrm{S} \mathrm{II}] / \mathrm{H} \alpha$ & $0.61(54)$ & $0.24(13)$ & $0.26(43)$ & $0.23(74)$ & $0.28(80)$ & $0.33(81)$ \\
\hline$I(6716) / I(6731)$ & $0.95(30)$ & $1.1(7)$ & $1.09(21)$ & $0.97(37)$ & $0.99(41)$ & $0.99(40)$ \\
\hline
\end{tabular}

${ }^{a}$ Fluxes uncorrected for interstellar extinction and relative to $F(\mathrm{H} \alpha)=100$.

${ }^{b}$ Listed fluxes are a signal to noise weighted average of the individual fluxes.

${ }^{c}$ In units of $10^{-17} \mathrm{erg} \mathrm{s}^{-1} \mathrm{~cm}^{-2} \operatorname{arcsec}^{-2}$.

Numbers in parentheses represent the signal to noise ratio of the quoted fluxes. The $1 \sigma$ error is given in square brackets for the logarithmic extinction. 


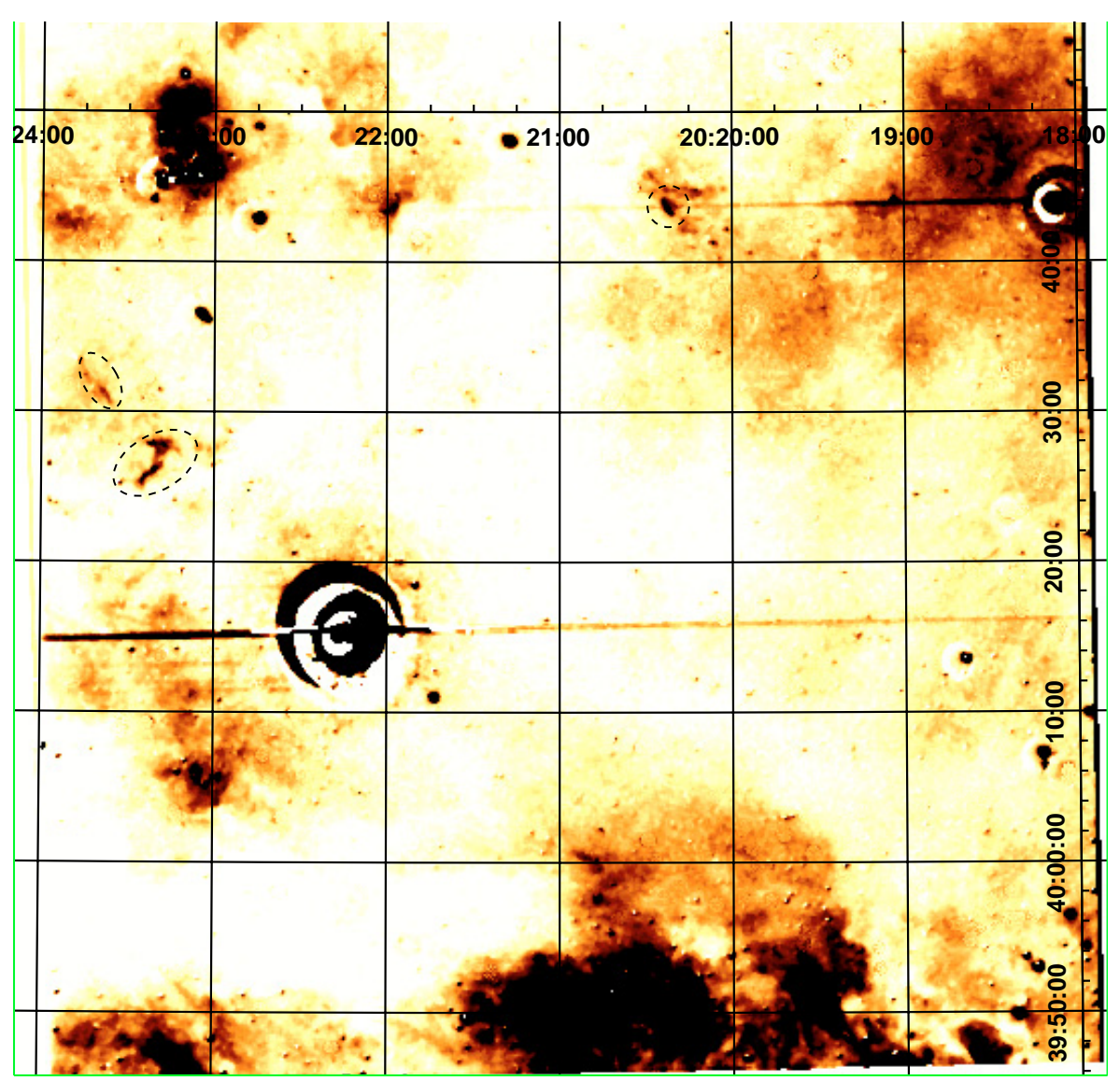

Fig. 3. The emission in the medium ionization line of [O III] $5007 \AA$ is shown in this figure. The dashed lines mark the detected small scale structures in the east and north-west. The shadings run linearly from 0 to $20 \times 10^{-17} \mathrm{erg} \mathrm{s}^{-1} \mathrm{~cm}^{-2} \mathrm{arcsec}^{-2}$.

recombination zone is $\sim 750 \mathrm{~cm}^{-3}$ as the sulfur lines ratio of 0.95 suggests. In addition, the $\mathrm{H} \alpha / \mathrm{H} \beta$ ratio is $\sim 10$, in contrast to the $\mathrm{H} \alpha / \mathrm{H} \beta$ ratio of $\sim 7$ measured in the second spectrum from the same area (pos. 2). The $\mathrm{H} \alpha / \mathrm{H} \beta$ ratio of 10 corresponds to a logarithmic extinction $\mathrm{c}$ of $1.6( \pm 0.2)$, adopting the interstellar extinction curve of Kaler (1976) as implemented in the redcorr task within the nebular package. The corresponding color excess $E(B-V)$ is then $1.1( \pm 0.2)$ assuming that $E(B-V)=0.664 \cdot c$ (Kaler 1976; Aller 1984). We can now calculate the hydrogen column density $N_{\mathrm{H}}$, using the statistical relation of Predehl \& Schmitt (1995). It is found that the equivalent hydrogen column density is $\sim 6( \pm 1) \times 10^{21} \mathrm{~cm}^{-2}$, which is substantially lower than the estimated total galactic $N_{\mathrm{H}}$ of $1.5 \times 10^{22} \mathrm{~cm}^{-2}$ (Dickey \& Lockmann 1990). We have also used the code of Hakkila et al. (1997) to estimate the color excess as a function of distance in the direction of G 78.2+2.1 and the following pairs of distance and color excess were obtained: $(1.0 \mathrm{kpc}, 0.8 \pm 0.2),(1.5 \mathrm{kpc}, 1.1 \pm 0.2),(2.0 \mathrm{kpc}, 1.4 \pm 0.3)$, $(2.5 \mathrm{kpc}, 1.6 \pm 0.3)$. Although the uncertainties are not negligible, these results show that our measurement of the color excess is compatible with the range of distances estimated for G 78.2+2.1 (Landecker et al. 1980). Note here that the signal to noise ratios given in Table 2 do not incorporate errors due to the calibration process which are $\sim 10 \%$.
Table 3. Spectral log.

\begin{tabular}{|c|c|c|}
\hline Slit centers & & Exp. times ${ }^{a}$ \\
\hline$\alpha$ & $\delta$ & $\left(\right.$ No of spectra) ${ }^{b}$ \\
\hline $20^{\mathrm{h}} 19^{\mathrm{m}} 17^{\mathrm{s}}$ & $40^{\circ} 45^{\prime} 20^{\prime \prime}$ & $7800(2)$ \\
\hline $20^{\mathrm{h}} 22^{\mathrm{m}} 53^{\mathrm{s}}$ & $40^{\circ} 26^{\prime} 21^{\prime \prime}$ & $7800(2)$ \\
\hline
\end{tabular}

\section{Discussion}

Earlier optical observations focused at the nebulosity in the immediate vicinity of $\gamma$ Cygni (e.g. d'Odorico \& Sabbadin 1977; van den Bergh 1978; Bohigas et al. 1983), although Higgs et al. (1977) had established the larger angular size of this remnant. In this work we present the first flux calibrated narrow band CCD images, covering the full extent of G 78.2+2.1, in the emission lines of $\mathrm{H} \alpha+[\mathrm{N}$ II], [S II], and [O III], together with deep long-slit spectra. The remnant lies in the Cygnus area along with $\mathrm{H}$ II regions, dark nebulae and clouds emitting strongly in the infrared as well as in molecular lines. Consequently, the search for optical emission associated with the remnant is a very difficult task. 
The low-ionization images of $\mathrm{H} \alpha+[\mathrm{N} \mathrm{II}]$ and [S II] display similar morphologies although the overall emission is somewhat suppressed in the latter image. Filamentary structures are not prominent and the diffuse emission is the major characteristic of the field of G 78.2+2.1. The [O III] emission line image reveals three elongated structures, one in the north-west and two in the east (Fig. 3). The low-ionization images provide evidence for emission from shock-heated gas for one of the structures in the east. This does not necessarily imply that the other two are not associated to $\mathrm{G} 78.2+2.1$, given the complexity of the environment, since they appear oriented along contours of radio emission. We have obtained estimates of the [S II]/H $\alpha$ ratio which reveal possible areas of emission from shock-heated gas. One of those is found in the south where diffuse emission dominates, while two filamentary structures in the east seem to be good candidates to associate with the remnant. Especially, the filaments in the east coincide with the intense filamentary $1400 \mathrm{MHz}$ emission in this area, while in the south the radio emission is not filamentary and overlaps the diffuse optical emission. We were able to show, through long-slit spectroscopy, that a faint filament in the east displays a [S II]/H $\alpha$ ratio typical to supernova remnants (Sect. 4; Table 2). The low $[\mathrm{O} \mathrm{III}] / \mathrm{H} \beta$ ratio suggests low shock velocities, probably below $90 \mathrm{~km} \mathrm{~s}^{-1}$ (e.g. Cox \& Raymond 1985). At these shock velocities the helium is basically neutral, while hydrogen can be $\sim 50 \%$ ionized, assuming preshock ionization equilibrium (e.g. Hartigan et al. 1987). The [O I] flux, amounting to only $\sim 5 \%$ of the $\mathrm{H} \alpha$ flux, can be accounted for by the low shock velocity. Furthermore, the weak [O III] emission in this part of $\mathrm{G} 78.2+2.1$ suggests complete recombination zones, i.e. the post-shock gas is able to cool down to very low temperatures (e.g. Raymond et al. 1988). The measured electron density of $\sim 750 \mathrm{~cm}^{-3}$, at the specific location, allows us to estimate a preshock cloud density of the order of $20 \mathrm{~cm}^{-3}$, in case of negligible magnetic field (Fesen \& Kirshner 1980). The $\mathrm{H} \alpha / \mathrm{H} \beta$ ratio of $10.2( \pm 1.7)$ is the highest measured in all spectra and may indicate that the $\mathrm{H}$ II regions are closer to us and seen in projection against the emission from the supernova remnant. Although a statistical nature of this difference cannot be excluded, we note that all four spectra from pos. 1 and the second spectrum from pos. 2 have systematically lower $\mathrm{H} \alpha / \mathrm{H} \beta$ ratios.

Saken et al. (1992) reported the detection of a partial shell in the IR using data from the IRAS satellite. However, the moderate resolution of these data $\left(\sim 2^{\prime}\right)$ does not permit a detailed comparison with the optical and radio data. New IR data were obtained by the Midcourse Space Experiment (MSX) in four bands and are of sufficient resolution $\left(\sim 20^{\prime \prime}\right)$ to compare with the optical and radio images. Substantial IR emission is present to the south and to the east of $\gamma$ Cygni (Fig. 2). Weaker emission extends further to west, north-west characterized by several patchy structures which seem to have the same curvature as the radio emission, while the central and north parts of the remnant are practically free of IR emission. Interestingly, the location of pos. 2 and its neighbor location are areas where the IR emission is, at least, a factor of 5 lower than that measured close to $\gamma$ Cygni. This may indicate that the dust clouds are in the foreground of the remnant possibly obscuring optical emission from these areas that otherwise would have been

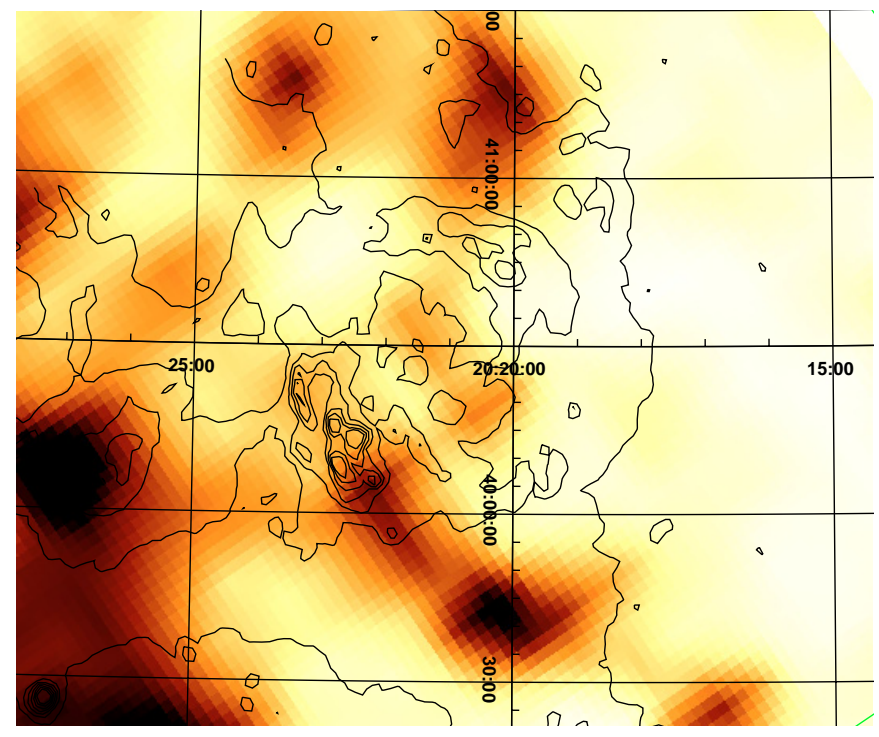

Fig. 4. The total molecular $\mathrm{CO}$ emission in the field of $\mathrm{G} 78.2+2.1$ with contours of the radio emission at $1400 \mathrm{MHz}$. Weak CO emission is mainly seen in the north-east, the west and towards the central

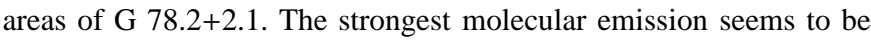
projected to the south-east of $\gamma \mathrm{Cygni}$. The $\mathrm{CO}$ data were interpolated to produce a smoother image.

detected. Diffuse infrared emission is present in the south where the morphology of the [S II] emission, that could be related to $\mathrm{G} 78.2+2.1$, is also diffuse.

The column density $N_{\mathrm{H}}$ is a measure of the absorption towards a source and is mainly determined through X-ray observations. The overall difference between the optical and X-ray determined column density can be accounted for by the presence of molecular gas which mainly affects the X-rays (see also Fesen \& Hurford 1995). Examination of CO emission maps (Fig. 4) shows that the equivalent molecular column density in the direction of G 78.2+2.1 is $\sim 2 \times 10^{21} \mathrm{~cm}^{-2}$ (Dame et al. 2001; Leung \& Thaddeus 1992). Although, the spatial resolution of this survey is $\sim 8^{\prime}$, we can measure a column density of $\sim 3 \times 10^{21} \mathrm{~cm}^{-2}$ at our pos. 2 , while the minimum of $\sim 0.5 \times 10^{21} \mathrm{~cm}^{-2}$ is found in the west, in the neighborhood of our pos. 1. The median value of the column density over the entire remnant is $\sim 2 \times 10^{21} \mathrm{~cm}^{-2}$ with the maximum of $\sim 7 \times 10^{21} \mathrm{~cm}^{-2}$ measured in a single bin to the south-east of $\gamma$ Cygni. We have also examined the velocity resolved neutral hydrogen maps of Hartmann \& Burton (1997). A column density of $\sim 6 \times 10^{21} \mathrm{~cm}^{-2}$ is measured in the direction of this remnant and for local standard of rest velocities appropriate to the Local Arm. Thus, the average total column density should not be more than $\sim 8 \times 10^{21} \mathrm{~cm}^{-2}$ according to these surveys. The average neutral hydrogen density of the interstellar medium towards $G 78.2+2.1$, and for a distance of $1.5 \mathrm{kpc}$, is a factor of $\sim 5$ higher than the ambient density of $\sim 0.2-0.3 \mathrm{~cm}^{-3}$ implied by X-ray and infrared observations. These densities are not unreasonable (see also Wendker et al. 1991; Landecker et al. 1980), if we realize that the remnant lies $\sim 55 \mathrm{pc}$ above the galactic plane, and may imply that most of the absorption the X-rays suffer, takes place in the 
foreground. The remnant evolves in a medium of low density, typically $\sim 0.3 \mathrm{~cm}^{-3}$, encompassing interstellar "clouds" of higher densities $\left(\sim 20 \mathrm{~cm}^{-3}\right)$. These clouds are responsible for the optical emission and a typical projected length scale is $\sim 0.7 \mathrm{pc}$. The density contrast of $\sim 70$ under the assumption of pressure equilibrium between the cloud and intercloud regions, implies a primary shock velocity of $\sim 750 \mathrm{~km} \mathrm{~s}^{-1}$ which agrees, within the errors, with the lower temperature measured in the east by Uchiyama et al. (2002).

Adopting the formulae of Hailey \& Craig (1994 and references therein), we estimate an explosion energy of $1.1 \times$ $10^{51} \mathrm{erg}$ and an age of $\sim 7000 \mathrm{yr}$, for a radius of $15 \mathrm{pc}$ and an interstellar medium density of $\sim 0.3 \mathrm{~cm}^{-3}$ (Uchiyama et al. 2002; Lozinskaya et al. 2000). Note also that the Sedov-Taylor solution for $\mathrm{G} 78.2+2.1$ implies an E51/n $n_{\mathrm{o}} \sim 5$ for a radius of $15 \mathrm{pc}$ and a shock velocity $\sim 900 \mathrm{~km} \mathrm{~s}^{-1}$ (Lozinskaya et al. 2000; Uchiyama et al. 2002) independently of the actual age. Here E51 stands for the explosion energy in units of $10^{51} \mathrm{erg}$ and $n_{\mathrm{o}}$ is the density of the ambient interstellar medium. Thus higher densities would imply accordingly higher energies or vice versa. The above estimates of the explosion energy and age are in very good agreement with those obtained from X-ray measurements analyzed by the aforementioned authors considering the uncertainties involved and the assumptions made. According to these calculations, shell formation would occur at $\sim 72000$ yr (Cioffi et al. 1988). However, radiative losses will manifest themselves earlier at an age of $\sim 26000 \mathrm{yr}$ which is a lot larger than the current age of the remnant. It can also be shown that for a primary shock velocity around $800 \mathrm{~km} \mathrm{~s}^{-1}$, it is not possible to obtain realistic values of the energy and ISM density assuming the age of the pressure driven snowplow phase to be equal to the current age of the remnant. Thus, it seems that $\mathrm{G} 78.2+2.1$ is currently in the adiabatic phase of its evolution.

\section{Conclusions}

The morphology of the optical emission as seen in the low and medium ionization line images is mainly patchy. The flux calibrated images provide evidence for emission from shockheated gas in the south and east areas of the remnant where the infrared emission is weak or absent. Long-slit spectra indicate low shock velocities, moderate electron densities and sufficient interstellar extinction which is nevertheless compatible with the extinction towards the Local Arm. The interstellar "clouds" appear to be in pressure equilibrium with the X-ray emitting gas, while the Sedov-Taylor solution is satisfied by an explosion energy of $\sim 10^{51} \mathrm{erg}$ and an age of $\sim 7000 \mathrm{yr}$.

Acknowledgements. The author would like to thank N. Kylafis, T. Landecker, J. Papamastorakis and the referee for their insightful and helpful comments. Skinakas Observatory is a collaborative project of the University of Crete, the Foundation for Research and Technology-Hellas and the Max-Planck-Institut für Extraterrestrische Physik. This research made use of data products from the Midcourse Space Experiment. Processing of the data was funded by the Ballistic Missile Defense Organization with additional support from NASA Office of Space Science. This research has also made use of the NASA/IPAC Infrared Science Archive, which is operated by the Jet
Propulsion Laboratory, California Institute of Technology, under contract with the National Aeronautics and Space Administration. The data from the Canadian Galactic Plane Survey were obtained from the Canadian Astronomy Data Centre (where the author is a guest user) which is operated by the Herzberg Institute of Astrophysics of the National Research Council Canada.

\section{References}

Aller, L. H. 1984, Physics of thermal gaseous nebulae (D. Reidel Publishing Company)

Bohigas, J., Ruiz, M. T., Carrasco, L., Salas, L., \& Herrera, M. A. 1983, RMxAA, 8, 155

Brazier, K. T. S., Kanbach, G., Carraminana, A., Guichard, J., \& Merck, M. 1996, MNRAS, 281, 1033

Cioffi, D. F., McKee, C. F., \& Bertschinger, E. 1988, ApJ, 334, 252

Cox, D. P., \& Raymond, J. C. 1985, ApJ, 298, 651

Dame, T. M., Hartmann D., \& Thaddeus, P. 2001, ApJ, 547, 792

Dickey, J. M., \& Lockman, F. J., 1990, ARA\&A, 28, 215

d'Odorico, S., \& Sabbadin, F. 1977, A\&AS, 28, 439

Esposito, J. A., Hunter, S. D., Kanbach, G., \& Sreekumar, P. 1996, ApJ, 461, 820

Fesen, R. A., \& Kirshner, R. P. 1980, ApJ, 242, 1023

Fesen, R. A., \& Hurford, A. P. 1995, AJ, 110, 747

Gervais, S., \& St-Louis, N. 1999, AJ, 118, 2394

Hailey, C. J., \& Craig, W. W. 1994, ApJ, 434, 635

Hakkila, J., Myers, J. M., Stidham, B. J., \& Hartmann, D. H. 1997, AJ, 114, 2043

Hamuy, M., Walker, A. R., Suntzeff, N. B., et al. 1992, PASP, 104, 533

Hamuy, M., Suntzeff, N. B., Heathcote, S. R., et al. 1994, PASP, 106, 566

Hartmann, D., \& Burton, W. B. 1997, Atlas of Galactic Neutral Hydrogen (Cambridge University Press)

Hartigan, P., Raymond, J., \& Hartmann, L. 1987, ApJ, 316, 323

Higgs, L. A., Landecker, T. L., \& Roger, R. S. 1977, AJ, 82, 718

Hunter, D. A. 1992, ApJS, 79, 469

Johnson, H. M. 1974, ApJ, 194, 337

Kaler, J. B. 1976, ApJS, 31, 517

Landecker, T. L., Roger, R. S., \& Higgs, L. A. 1980, A\&AS, 39, 133

Lasker, B. M., Russel, J. N., \& Jenkner, H. 1999, in the HST Guide Star Catalog, version 1.1-ACT (The Association of Universities for Research in Astronomy, Inc.)

Lozinskaya, T. A., Pravdikova, V. V., \& Finoguenov, A. V. 2000, Astron. Let., 26, 77

Leung, H. O., \& Thaddeus, P. 1992, ApJS, 81, 267

McKee, C. F., \& Cowie, L. L. 1975, ApJ, 195, 715

Parker, R. A., Gull, T. R., \& Kirshner, R. P. 1979, An emission line survey of the Milky Way (Washington DC: NASA)

Pineault S., \& Chastenay P. 1990, MNRAS, 246, 169

Predehl, P., \& Schmitt, J. H. M. M. 1995, A\&A, 293, 889

Raymond, J. C., Hester, J. J., Cox, D., et al. 1988, ApJ, 324, 869

Saken, J. M., Fesen, R. A., \& Shull, J. M. 1992, ApJS, 81, 715

Shaw, R. A., \& Dufour, R. J. 1995, PASP, 107, 896

Smith, R. C., Kirshner, R. P., Blair, W. P., Long, K. S., \& Winkler, P. F. 1993, ApJ, 407, 564

Taylor, A. R., Gibson, S. J., Peracaula, M., et al. 2003, AJ, 125, 3145

Uchiyama, Y., Takahashi, T., Aharonian, F. A., \& Mattox J. R. 2002, ApJ, 571, 866

van den Bergh, S. 1978, ApJS, 38, 119

Wendker, H. J., Higgs, L. A., Landecker, T. L. 1991, A\&A, 241, 551

Zhang, Z., Zheng, Y., Landecker, T. L., \& Higgs L. A. 1997, A\&A, 324,641 